\title{
Global univalence when mappings are not necessarily continuous*
}

\author{
Taradas Bandyopadhyay \\ University of California, Riverside CA, USA
}

\section{Tapan Biswas}

University of Hull, Hull, UK

Submitted November 1989, accepted September 1992

\begin{abstract}
This paper proposes a method of establishing the global univalence of a mapping without the assumption of continuity and the absence of points of inflection. When the functions are not continuous and the points of inflections are present, the use of a Jacobian to establish univalence presents some difficulties. The method of establishing univalency, presented in this paper, in turn generalizes the theorems on the uniqueness of competitive equilibrium and factor price equalization.
\end{abstract}

Key words: Continuity; Univalence; Dominance; Equilibrium JEL classifications: C60; D50

\section{Introduction}

The study of univalent mappings has been considered in economics in the context of establishing the uniqueness of competitive equilibrium, the factorprice equalization theorem and the generalized Leontief system. Traditionally, the univalence of a mapping is ensured by examining the Jacobian matrix which requires the mapping to be differentiable, e.g. the Jacobian is to be a P- (or NP-) matrix [Nikaido (1968)] or quasi-dominant diagonal matrix [McKenzie (1959)] or it should satisfy related restrictions [Mas-Colell (1970, 1979, 1985), Pearce and Wise (1973, 1974), Kehoe (1985)]. In an interesting development of the literature on univalency, Mas-Colell and Kehoe [see Mas-Colell (1985)] developed the 'index theory' using a bordered Jacobian. However, like others' works mentioned above, their approach is also

Correspondence to: Taradas Bandyopadhyay, Department of Economics, University of California, Riverside, CA 92521, USA.

*Thanks to an editor of this journal for helpful comments and suggestions. The usual disclaimer, of course, applies. 
restricted to the analysis of smooth economies with differentiable excess demand functions. The differentiability of a mapping is indeed a stringent assumption when we recall that in order to prove the existence of a competitive equilibrium we take pains to avoid the assumption of continuity of excess demand correspondence [Arrow and Hahn (1971)]. Considering the initial endowment of an individual at the boundary of the consumption set, it is easy to construct a simple example to see the possibility of discontinuity in the (excess) demand correspondence. ${ }^{1}$ In the context of the factor-price equalization theorem, the unit cost functions are not necessarily differentiable everywhere in the domain (e.g., when the unit isoquant is the convex combination of a set of discrete points). Hence, the study of univalency without the assumptions of continuity and differentiability is of considerable significance in economics. In the absence of continuity, the Jacobian techniques do not work in examining univalency. ${ }^{2}$ Hence, in the absence of continuity we need an alternative approach to establish the univalency of a mapping. Furthermore, in all results on global univalence, the conditions imposed on the Jacobian imply that the Jacobian and all its principal minors are bounded away from zero. Vanishing of the Jacobian does not necessarily imply degenerate mapping (absence of local homeomorphism). It may happen at points of inflection, which is admissible under univalent mapping. ${ }^{3}$ Apart from inflection, there is another interesting case where vanishing Jacobians may be associated with univalent mappings. For a better intuitive understanding, we shall discuss this case in section 5 .

\section{The preliminaries}

We shall be working in the real space $\mathbb{R}^{n}$; the representation of dimensionality $n$ of the space depends on the economic models. For example, $x \in \mathbb{R}^{n}$ represents factor prices in the context of the factor-price equalization theorem and commodity prices in the context of competitive equilibrium.

${ }^{1}$ For the definition of consumption set, see Arrow and Hahn (1971). In economic literature, discontinuous functions are typically associated with correspondences and, in particular, upper semi-continuous correspondences. One important aspect of our approach is that it can deal with such complexities.

${ }^{2}$ For the sake of a simple illustration, consider a function $y=f(x)$ such that

$$
\begin{aligned}
& y=60+7 x \text { for } 0 \leq x<20 \text {, } \\
& y=4 x \text { for } 20 \leq x<\infty .
\end{aligned}
$$

The function $f(x)$ has positive derivatives almost everywhere inside the domain (hence, trivially satisfies the P-matrix criterion) except al $x=20$ where the discontinuity occurs. Although the Pmatrix criterion is satisfied almost everywhere, the function is not univalent (e.g., for $y=172$, there are two solutions $x=16$ and $x=43$ ).

${ }^{3}$ The points of inflection alone do not create much problem. In fact, it is trivial to perturb away inflection points of univalent mappings. Furthermore, in certain cases, the study of univalency involving nondifferentiable mappings could be handled by adopting the technique of nonsmooth analysis [see Hiriar-Urruty $(1981,1982)]$.

Originally published in Journal of Mathematical Economics (1994) 23(5): 435-450. 
Following standard practice, for $x$ and $x^{\prime}$ in $\mathbb{R}^{n}$ the notation $x^{\prime} \geqq x$ implies $x_{i}^{\prime} \geq x_{i}$ for all $i, i \geq 1, \ldots, n$. The notation $x^{\prime} \geq x$ implies $x_{i}^{\prime} \geq x_{i}$ for all $i$ and $x_{i}^{\prime}>x_{i}$ for some $i$. Let $\Omega$ denote the nonnegative orthant $\mathbb{R}^{n}$. We are interested in the following set of equations:

$$
y_{i}=f_{i}\left(x_{i}, \ldots, x_{n}\right), \quad i=1,2, \ldots, n .
$$

In section 3 , we assume $x, y \in \Omega$, where we discuss the case of nondecreasing functions. However, the main theorem in this section has been stated in a more general context. In terms of economic interpretation, this case is related to the factor-price equalization theorem where the factor prices and the commodity prices are nonnegative. On the other hand in section 4 , both the domain and the range of the mapping $y=f(x)$ are assumed to be unrestricted. Again in terms of economic interpretation, this section is related to the study of uniqueness of competitive equilibrium. Excess demands may be either negative or positive. Similarly, prices may be negative if we do not allow for free disposability. If we assume that commodities are freely disposable, then the prices $(x)$ are nonnegative. The theorems proved in section 4 may also be extended to this case using logarithmic transformation of variables. Note, (i) $\log y, \log x \in \mathbb{R}^{n}$ where $x, y \in \Omega$, (ii) if $y$ is an increasing function in $x$, it will also be an increasing function in $\log x$.

Definition 1. Let $A=\left[a_{i j}\right]$ be a real $n \times n$ matrix. $A$ is said to be

(1.1) Dominant diagonal if $\left|a_{j j}\right|>\sum_{i \neq j}\left|a_{i j}\right|$ for each $j$.

(1.2) Generalized dominant diagonal if there exists a number, $d_{j}>0, j=$ $1,2, \ldots, n$ such that $d_{j}\left|a_{j j}\right|>\sum_{i \neq j} d_{i}\left|a_{i j}\right|$.

(1.3) Quasi-dominant diagonal if there exists $d_{j}>0$, such that $d_{j}\left|a_{j j}\right| \geq \sum_{i \neq j} d_{i}$ $\left|a_{i j}\right|(j=1, \ldots, n)$, and when $a_{i j}=0$ (given $j \in J$ and $i \neq j$ for some set of indices $J$ ), the strict inequality holds for some $j \in J$.

(1.4) $P$-matrix if all principle minors are positive.

(1.5) NP-matrix if all principle minors of odd orders are negative and those of even orders are positive.

(1.6) Weak gross substitute (WGS) if $a_{j j} \leq 0$ and $a_{i j} \geq 0, i \neq j, i, j=1,2, \ldots, n$.

McKenzie (1959, Corollary of Theorem 4) has established that any square matrix which is quasi-dominant diagonal is also a generalized dominant diagonal matrix. The importance of the various dominant diagonal matrices lies in their applications in a number of economic problems. A matrix having positive (negative) dominant diagonal is a $\mathbf{P}$ - (NP-) matrix. In general a $\mathbf{P}$ or NP-matrix is not a quasi-dominant (or generalized dominant) diagonal matrix. However, if a matrix has a specific sign-pattern, e.g. nonnegative (or WGS) sign pattern, then a P- (or NP-) matrix is a quasi-dominant diagonal matrix. In economics, quite often we are concerned with a Jacobian matrix whose sign structure is known. Two such frequently discussed signed-

Originally published in Journal of Mathematical Economics (1994) 23(5): 435-450. 
matrices are (i) nonnegative matrices and (ii) WGS matrices. For example, the Jacobian of the unit cost functions or the Jacobian of the input-output functions is a nonnegative matrix. In general equilibrium theory, the case of weak gross substitutes yields a Jacobian of the excess demand functions which is a WGS matrix. However, as we have remarked earlier, the assumption that the underlying functions are continuous or the principal minors are bounded away from zero, imposes some severe restrictions. We shall now explain why the univalency of a mapping may be established without such restrictions by presenting an alternative approach.

Let us first investigate some implications of the dominant diagonal property which would provide an insight for the alternative approach. Let a set of functions (1) be differentiable and increasing with respect to all its arguments, i.e., $f_{i j}=\delta y_{i} / \delta x_{j}>0$ for all $i$ and $j$. The Jacobian is obviously a positive matrix. The above equations may alternatively be written as

$$
y_{i}=f_{i}\left(\left\{\left(x_{1}+x_{i}\right)-x_{i}\right\}, \ldots, x_{i}, \ldots,\left\{\left(x_{n}+x_{i}\right)-x_{i}\right\}\right)=P_{i}\left(t_{1 i}, \ldots t_{i i}, \ldots t_{n i}\right),
$$

where $t_{j i}=x_{j}+x_{i}, i \neq j$ and $t_{i i}=x_{i}$. Note, $T=\left(t_{j i}\right) \in \Omega$ for $x \in \Omega$. Clearly, $p_{i j}=$ $\left(\delta P_{i} / \delta t_{j i}\right)=\left(\delta f_{i} / \delta x_{j}\right)>0, i \neq j ;$ and $p_{i i}=\left(\delta P_{i} / \delta t_{i i}\right)=\left[\left(\delta f_{i} / \delta x_{i}\right)-\sum\left(\delta f_{i} / \delta x_{j}\right)\right]>0$ if the Jacobian $\left[f_{i j}\right]$ is a positive dominant diagonal matrix. It is now obvious that the positive dominant diagonal property of the Jacobian of a mapping $y=f(x)$, where $x, y \in \Omega$, implies that the transformed functions $y_{i}=P_{i}\left(t_{1 i}, \ldots, t_{n i}\right), i=1,2, \ldots, n$, have positive partial derivatives, $\left(\delta y_{i} / \delta t_{j i}\right)>0$, for all $i$ and $j$.

This observation on the positive dominant diagonal property suggests to our intuition that for a certain class of mappings (e.g. when the mapping is a set of nondecreasing functions), the continuity assumption is not necessary. We may replace the $\delta y_{i} / \delta \iota_{j i} \geqq 0$ with the property that $y_{i}$ is a nondecreasing function in $t_{j i}(j=1, \ldots, n)$ even allowing for 'jumps'.

\section{Univalency: The case of nondecreasing mappings}

A mapping $y=f(x)$ is said to be univalent if for any assigned $y$-vector the solution vector $x$ is unique. The function $f_{i}$ is increasing with respect to $x_{j}$ if $f_{i}\left(x_{1}^{\prime}, \ldots, x_{n}^{\prime}\right)>f_{i}\left(x_{1}, \ldots, x_{n}\right)$ for $x_{j}^{\prime}>x_{j}$ and $x_{i}^{\prime}=x_{i}$ for all $i \neq j$. The function is said to be nondecreasing if the strict inequality of $f_{i}$ is replaced by weak inequality.

For a set of increasing functions the positive dominant diagonal property of the Jacobian implies that a simultaneous increase in the $i$ th variable and decrease of same magnitude in all other variables increases the value of the $i$ th function. To capture the essence of this argument for nondecreasing functions which are not necessarily continuous, it is instructive to introduce a transformation function.

Originally published in Journal of Mathematical Economics (1994) 23(5): 435-450. 
Definition 2. Given $y_{i}=f_{i}\left(x_{i}, \ldots, x_{n}\right), i=1,2, \ldots, n$, a function $P_{i}$ is defined for $t_{j i}=x_{j}+x_{i}, i \neq j$ and $t_{i i}=x_{i}$ as

$$
P_{i}\left(t_{1 i}, \ldots, t_{i i}, \ldots, t_{n i}\right)=f_{i}\left(\left(t_{1 i}-t_{i i}\right), \ldots, t_{i i}, \ldots,\left(t_{n i}-t_{i i}\right)\right) \text {, }
$$

where $x \in \Omega$ and $T=\left(t_{j i}\right) \in \Omega$.

Although the effect of a change in $x_{j}$ on $P_{i}$ and $f_{i}$, where $i \neq j$, are the same, any change in $x_{i}$ alone affects all other arguments of the $P_{i}$ function. Now to examine the effect of an increase in only the $i$ th argument of $P_{i}$ one must simultaneously increase $x_{i}$ and decrease all other $x_{j}$ 's by the same amount.

Definition 3. Let $P_{i j}$ denote $P_{i}$ as a function of $t_{j i}$. The function $f_{i}$ is said to be

(3.1) nonnegatively responsive iff $P_{i i}$ is nondecreasing;

(3.2) positively responsive iff $P_{i i}$ is increasing;

(3.3) sensitive to $x_{j}$ iff $P_{i j}$ is increasing.

Positive responsiveness (respectively nonnegative responsiveness) requires that a simultaneous increase in $x_{i}$ and decrease in all other $x_{j}$ 's increases (respectively does not change) the value of $f_{i}$. Sensitivity to $x_{j}$ says that an increase in $x_{j}$ results in an increase in $f_{i}$.

Definition 4. Let $y=f(x), x, y \in \Omega$, be a set of nondecreasing functions. Let $P_{i}$ and $P_{i j}$ be defined for $i, j=1, \ldots, n$. Then $f$ satisfies the weakly positive dominance condition (WPD) iff for every $i=1, \ldots, n, f_{i}$ is nonnegatively responsive and there is a sequence $i(0), i(1), \ldots, i(k)$, where $i(0)=i$, such that for $s=0,1, \ldots, k-1, f_{i(s)}$ is sensitive to $x_{i(s+1)}$ and $f_{i(k)}$ is positively responsive.

Note that for every $i=1, \ldots, n$, if $f_{i}$ is positively responsive then WPD is trivially satisfied. In other words, WPD requires that for every $i=1, \ldots, n$ either $P_{i i}$ is increasing or $P_{i i}$ is nondecreasing and there is a sequence $i(0)$, $i(1), \ldots, i(k)$, where $i(0)=i$, such that for $s=0,1, \ldots, k-1, P_{i(s) i(s+1)}$ and $P_{i(k) i(k)}$ are increasing. ${ }^{4}$

For an economic interpretation, suppose that $n$ equations given in (1) is a set of unit cost functions where $y_{i}$ is the price of commodity $i$ and $x_{j}$ is the price of factor $j$. Now the nonnegative responsiveness (i.e., nondecreasing $P_{i i}$ ) requires that both the goods and the factors may be ranked in such a way that an increase in $x_{i}$ and an equal reduction in all other $x_{j}$ 's will not reduce $y_{i}$. The chain condition of WPD may be interpreted in the following way: for any $j$ in the index set, either the industry $j$ is positively responsive to the factor $j$ or there exists a sequence $j(0), j(1), \ldots, j(k)$ with $j(0)=j$ such that the industry $j(s)$ is sensitive to the price of factor $j(s+1)$ for $s=0,1, \ldots, k-1$

\footnotetext{
${ }^{4}$ The origin of a related type of mapping can be found in network theory. See Duffin (1948) where he considered continuous mapping in proving the existence and uniqueness of a solution.
}

Originally published in Journal of Mathematical Economics (1994) 23(5): 435-450. 
and the industry $j(k)$ is positively responsive to the factor $j(k)$. In a two-good, two-factor world WPD is trivially satisfied whenever goods and factors are ranked in such a way that the price of each good is positively responsive to the price of a distinct factor.

Consider an $n \times \mathrm{n}$ sign matrix which has $a$ ' + ' (or 0$)$ sign in the entry of $i$ th row and $j$ th column if $P_{i j}$ is increasing (or locally constant). The chain condition of WPD establishes the connection between the elements of the sign matirx which is constructed from the original mapping $y=f(x)$. Following is an example of a mapping which satisfies WPD but its Jacobian is neither a $P$-matrix nor a positive dominant diagonal matrix. ${ }^{5}$

Example 1. Consider the following set of functions:

$$
\begin{aligned}
& y_{1}=\left(x_{1}-1\right)^{3}+2\left(x_{1}+x_{2}\right), \\
& y_{2}=x_{1}+x_{2}, \\
& y_{3}=x_{2}+x_{3} .
\end{aligned}
$$

It is easy to check that the Jacobian of (2) vanishes whenever $x_{1}=1$; and the Jacobian matrix of (2) is neither a P-matrix nor a positive dominant diagonal matrix for all $x \in \Omega$. Obviously, given any $\left(y_{1}, y_{2}, y_{3}\right) \geqq 0$, if any $x=\left(x_{1}, x_{2}, x_{3}\right)$ satisfies (2), then $x$ is unique. The system of eqs. (2) is a simple illustration of a univalent mapping whose univalency cannot be established by using the P-matrix or the dominant diagonal matrix technique. However, it is easy to see (2) satisfies WPD. Consider the matrix of the $P_{i j}$ functions which are nondecreasing. Use the symbol $(+)$ when $P_{i j}$ is an increasing function and $(0)$ otherwise. The matrix $\left[p_{i j}\right]$ which corresponds to our illustration has the following sign-pattern:

$$
\left[p_{i j}\right]=\left[\begin{array}{ccc}
(+) & (+) & (0) \\
(+) & (0) & (0) \\
(0) & (+) & (0)
\end{array}\right] \text {. }
$$

Note, $P_{11}$ is an increasing function in $x_{1}$ even at $x_{1}=1$ although the derivative of $\left(x_{1}-1\right)^{3}$ with respect to $x_{1}$ vanishes whenever $x_{1}=1$. From the sign-pattern of the elements in (3) it is obvious that (2) satisfies WPD.

Although, in the economic literature, we generally consider the domain of a mapping to be either $\mathbb{R}^{n}$ or $\Omega$, we shall state the main result of this section in a generalized form. Let $S$ be defined as a positively comprehensive set if

\footnotetext{
${ }^{5}$ The same point can be made trivially with the mapping $y=x^{3}$. Our example illustrates the construction of $\left[p_{i j}\right]$ and the use of the chain condition for WPD.
}

Originally published in Journal of Mathematical Economics (1994) 23(5): 435-450. 
$S+\Omega \subset S .^{6}$ Now assuming that $S$ is a positively comprehensive set, we present the main result of this section:

Theorem 1. Let $y=f(x), x, y \in S$, be a set of nondecreasing functions satisfying WPD. For any assigned vector $y$ if a solution vector $x$ exists then $x$ is unique.

This result can be extended to semicontinuous correspondences with some suitable definitions for increasing and decreasing correspondences. Define a correspondence $F_{i}$ to be increasing in $x_{j}$ if $y_{i}^{\prime}>y_{i}$ for any $y_{i}^{\prime} \in F_{i}$ $\left(x_{1}, \ldots, x_{j-1}, x_{j}^{\prime}, x_{j+1}, \ldots, x_{n}\right)$ and any $y_{i} \in F_{i}\left(x_{1}, \ldots, x_{n}\right)$ where $x_{j}^{\prime}>x_{j}$. We define a correspondence $F_{i}$ to be nondecreasing with respect to $x_{j}$ in a similar way. The reader should note that if $F_{i}$ is nondecreasing in its arguments, then the 'graph' of the correspondence is 'thin' (single valued $y_{i}$ ), almost everywhere in the domian of $x_{j}$. Intuitively, it should be clear, that if the graph were not thin almost everywhere, we would have a problem in having any reasonable theorem on univalence.

If $F_{i}(x)$ is a nondecreasing correspondence, then construct a set of derived funtions $y=f(x)$ choosing any arbitrary $y_{i} \in F_{i}$ where $F_{i}$ is multivalued. The set of functions $y=f(x)$ is a set of nondecreasing functions (not necessarily continuous). It is clear that if $F(x)$ satisfies WPD, so does $f(x)$. Now suppose $F(x)$ satisfies WPD and for some $y \in F(x)$, there exists $x, x^{\prime}$ such that $y=F(x)=F\left(x^{\prime}\right)$. It is then possible to construct a derived function $f(x)$ - not necessarily continuous - such that $y=f(x)=f\left(\mathrm{x}^{\prime}\right)$. But we know that a derived function of $F(x)$ must also satisfy WPD and by Theorem 1, $f(x) \neq f\left(x^{\prime}\right)$. Therefore, $F(x)$ must be univalent. A similar kind of extension to Theorem 2 in section 4 can easily be constructed.

It is obvious that if $f(x), x, y \in S$, is differentiable everywhere and if its Jacobian is a nonnegative matrix which has the property of having a positive dominant diagonal, i.e., $p_{i i}=\left(f_{i i}-\sum_{i \neq j} f_{i j}\right)>0$ for every $i=1, \ldots, n$, then $y=f(x)$ must satisfy WPD. Example 1 shows that the converse is not true. In other words, the requirement of WPD, like $P$-matrix and quasi-dominant diagonal matrix, is weaker than the requirement of positive dominant diagonal property of the Jacobian matrix. Furthermore, as Example 1 shows, the requirement of P-matrix sometime may appear to be more demanding than necessary for univalency of a mapping.

To see the relationship between the quasi-dominant diagonal property of the Jacobian and WPD, let us define variables $x_{i}^{*}$ such that $d_{i}\left(x_{i}^{*}\right)=x_{i}$ with derivative $d_{i}^{\prime}>0, i=1, \ldots, n$. Apparently, $d_{i}^{\prime} \delta x_{i}^{*}=\delta x_{i}$. As before one may define $t_{j i}^{*}=x_{i}^{*}+x_{j}^{*}$ and $t_{i i}^{*}=x_{i}^{*}$ that allows us to write $y_{i}=p_{i}^{*}\left(t_{1 i}^{*}, \ldots, t_{n i}^{*}\right)$. Now $p_{i j}^{*}=\delta P_{i}^{*} / \delta t_{j i}^{*}=\delta y_{i} / \delta x_{j}^{*}=d_{j}^{\prime}\left(\delta f_{i} / \delta x_{j}\right)$ and $p_{i i}^{*}=\delta P_{i}^{*} / \delta t_{i i}^{*}=\delta y_{i} / \delta x_{i}^{*}-\sum_{j \neq i} \delta y_{i} / \delta x_{j}^{*}=$

\footnotetext{
${ }^{6}$ The comprehensive set in Levhari et al. (1975) may be regarded as a negatively comprehensive set.
}

Originally published in Journal of Mathematical Economics (1994) 23(5): 435-450. 
$d_{i}^{\prime}\left(\delta f_{i} / \delta x_{i}\right)-\sum_{j \neq i} d_{j}^{\prime}\left(\delta f_{i} / \delta x_{j}\right)$. If the Jacobian of a mapping $y=f(x)$ has a positive generalized dominant diagonal with the choice of weights as $d_{1}^{\prime}, d_{2}^{\prime}, \ldots, d_{n}^{\prime}$, then $p_{i i}^{*}>0$. It is clear that the positive generalized dominant diagonal property of the mapping $y=f(x), x, y \in S$, implies that the transformed function $y_{i}=p_{i}^{*}\left(t_{1 i}^{*}, \ldots, t_{n i}^{*}\right)$ has positive partial derivatives, $\delta y_{i} / \delta t_{j i}^{*}>0$ for all $i, j$ and $\left(t_{j i}^{*}\right) \in S$. Now we make the following remark.

Remark 1. Let $y=f(x), x, y \in S$, be a set of nondecreasing and differentiable functions. If the Jacobian of $f(x)$ is a positive generalized quasi-dominant diagonal matrix with weights $d_{i}>0(i=1, \ldots, n)$, then there exist variables $x_{i}^{*}$, where $x_{i}=d_{i} x_{i}^{*}(i=1, \ldots, n)$, such that $y=g\left(x^{*}\right)$ satisfies WPD. The converse is not true.

Proof. Since we are dealing with square matrices, by McKenzie's corollary [13], a quasi-dominant diagonal matrix is also a generalized dominant diagonal matrix. Therefore, we have to show that if the Jacobian of $y=f(x)$ is a positive generalized dominant diagonal then $y=g\left(x^{*}\right)$ satisfies WPD.

Define $t_{i i}^{*}=x_{j}^{*}+x_{i}^{*}=x_{i} / d_{i}+x_{j} / d_{j}$ and $t_{i i}^{*}=x_{i}^{*}=x_{i} / d_{i}$. Then $\delta P_{i}^{*} / \delta t_{j i}^{*}=$ $\delta y_{i} / \delta x_{j}^{*}=d_{j}\left(\delta y_{i} / \delta x_{j}\right) \geqq 0$, and $\delta P_{i}^{*} / \delta t_{i i}^{*}=\delta y_{i} / \delta x_{i}^{*}-\sum_{j \neq i} \delta y_{i} / \delta x_{j}^{*}=d_{i}\left(\delta y_{i} / \delta x_{j}\right)-$ $\sum_{j \neq i} d_{j}\left(\delta y_{i} / \delta x_{j}\right)>0$. Since the Jacobian of $y=f(x)$ satisfies the positive generalized dominant diagonal property, the above implies that $y$ expressed as a function of $x^{*}$ satisfies WPD trivially (i.e., we do not have to invoke the chain condition of WPD).

Example 1 shows that the converse is not true. Note, the Jacobian of the set of functions of the example remains a singular matrix for any strictly monotonic transformation of the variable $x$.

\section{Univalency: The case of nonincreasing mappings}

So far we have considered the nondecreasing functions and have established the relationship with the nonnegative Jacobian matrices used in the international trade theory and in the generalized Leontief model. In the context of competitive equilibrium analysis, the mappings we encounter, i.e., the excess demand (supply) functions, are neither nondecreasing nor nonincreasing with respect to all its arguments. In the well-known case of 'weak gross substitutes' (WGS), the excess demand functions are nonincreasing with respect to own-price and nondecreasing with respect to other prices.

Let $y_{i}=f_{i}\left(x_{i}, \ldots, x_{n}\right), i=1, \ldots, n$. Throughout this section we assume that $f_{i}$ is nonincreasing with respect to $x_{i}$ and nondecreasing with respect to $x_{j}$, $j \neq i$. To deal with this class of mappings, it is instructive to introduce the following transformation function.

Definition 5. Given $y_{i}=f_{i}\left(x_{1}, \ldots, x_{n}\right), i=1,2, \ldots, n$, a new function $R_{i}$, is Originally published in Journal of Mathematical Economics (1994) 23(5): 435-450. 
defined for $t_{j i}=x_{i}-x_{j}, \quad i \neq j$ and $t_{i i}=x_{i}$ as $R_{i}\left(t_{1 i}, \ldots, t_{i i}, \ldots, t_{n i}\right)=$ $f_{i}\left(\left(t_{i i}-t_{1 i}\right), \ldots, t_{i i}, \ldots,\left(t_{i i}-t_{n i}\right)\right)$.

Definition 6. Let $R_{i j}$ denote $R_{i}$ as a function of $t_{j i}$. The function $f_{i}$ is said to be

(6.1) nonpositively responsive iff $R_{i i}$ is nonincreasing,

(6.2) negatively responsive iff $R_{i i}$ is decreasing,

(6.3) sensitive to $x_{j}$ iff $R_{i j}$ is decreasing.

Negative responsiveness (respectively nonpositive responsiveness) requires that a simultaneous increase in all $x_{i}^{\prime}$ s by the same amount decreases (respectively does not change) the value of $f_{i}$. Once again, sensitivity to $x_{j}$ says that an increase in $x_{j}$ results in an increase in $f_{i}$.

Definition 7. Let $y=f(x), x, y \in \mathbb{R}^{n}$, be a set of functions such that for each $i$, $f_{i}$ is nonincreasing in $x_{i}$ and nondecreasing in $x_{j}$ for all $j \neq i$. Let $R_{i}$ and $R_{i j}$ be defined for $i, j=1, \ldots, n$. Then $f$ satisfies the weakly negative dominance condition (WND) iff for every $i=1, \ldots, n, f_{i}$ is nonpositively responsive and there is a sequence $i(0), i(1), \ldots, i(k)$, where $i(0)=i$, such that for $s=$ $0,1, \ldots, k-1, f_{i(s)}$ is sensitive to $x_{i(s+1)}$ and $f_{i(k)}$ is negatively responsive.

Once again note that for every $i=1, \ldots, n$, if $f_{i}$ is negatively responsive then WND is trivially satisfied. In other words, the WND condition requires that for every $i=1, \ldots, n$ either $R_{i i}$ is decreasing or $R_{i i}$ is nonincreasing and there is a sequence $i(0), i(1), \ldots, i(k)$, where $i(0)=i$, such that for $s=0,1, \ldots, k-1$, $R_{i(s) i(s+1)}$ and $R_{i(k) i(k)}$ are decreasing. In the context of WND the following result is parallel to Theorem $1^{7}$

Theorem 2. Let $y=f(x), x, y \in \mathbb{R}^{n}$, be a set of functions such that for each $i, f_{i}$ is nonincreasing in $x_{i}$ and nondecreasing in $x_{j}$ for all $j \neq i$. Let $f$ satisfy $W N D$. For any assigned $y$ if a solution vector $x$ exists then $x$ is unique.

Our next theorem establishes that WND is both necessary and sufficient for global univalency of nondecreasing mappings which satisfy nonpositive responsiveness. This result in turn shows further the importance of chain relation that we have used in defining WND.

${ }^{7}$ Following the method of constructing $p_{\mathrm{ij}}$ 's from $p^{i}(\cdot)$, construct $r_{i j}$ 's from $R_{i}, i=1, \ldots, n$ such that

$$
\begin{aligned}
& 0=r_{11} \delta x_{1}+r_{12}\left(\delta x_{1}-\delta x_{2}\right)+\cdots+r_{1 n}\left(\delta x_{1}-\delta x_{n}\right), \\
& \vdots \\
& 0=r_{n 1}\left(\delta x_{n}-\delta x_{1}\right)+r_{n 2}\left(\delta x_{n}-\delta x_{2}\right)+\cdots+r_{n n} \delta x_{n 1},
\end{aligned}
$$

where, by WND, $r_{i j} \leqq 0$ for $i, j=1, \ldots, n$. The rest of the proof is very similar to Theorem 1 .

Originally published in Journal of Mathematical Economics (1994) 23(5): 435-450. 
Theorem 3. Let $y_{i}=f_{i}\left(x_{1}, \ldots, x_{n}\right), i=1, \ldots, n$, be a set a functions which are nonincreasing in $x_{i}$ and nondecreasing in $x_{j}$ for $j \neq i$. Let $f_{i}(i=1, \ldots, n)$ be nonpositively responsive. Then $y=f(x), x, y \in \mathbb{R}^{n}$, is univalent if and only if $f(x)$ satisfies WND.

Once again, following the proof of Remark 1, the observation below is immediate.

Remark 2. Let $y=f(x), x, y \in \mathbb{R}^{n}$ be a set of differentiable mappings whose Jacobian is a WGS. If the Jacobian of $f(x)$ is a negative quasi-dominant diagonal matrix with weights $d_{i}>0(i=1, \ldots, n)$, then there exist variables $x_{i}^{*}$, where $x_{i}^{*}=d_{i} x_{i}(i=1, \ldots, n)$, such that $y=g\left(x^{*}\right), x^{*} \in \mathbb{R}^{n}$, satisfies WND. The converse is not true.

In general a set of functions may not all be nondecreasing as required by WPD or satisfy a similar type of restriction required by WND. However, if these functions can be transformed to satisfy WPD and WND by any monotonic transformation of the variables, then univalency holds. For example let

$$
x_{i}=\Psi_{i}\left(q_{i}\right), \quad i=1,2, \ldots, n,
$$

such that $\Psi_{i}$ is strictly monotonic and unbounded. Substituting (4) in (1) we get

$$
y_{i}=f_{i}\left(\Psi_{1}\left(q_{1}\right), \ldots, \Psi_{n}\left(q_{n}\right)\right)=F_{i}\left(q_{1}, \ldots, q_{n}\right), \quad i=1,2, \ldots, n .
$$

Since (1) may not be continuous, ( $\left(1^{\prime}\right)$ is not necessarily continuous. Now the following observation is immediate.

Remark 3. If (1') satisfies WPD or WND then (1) is a univalent mapping.

As explained in section 3, Theorems 2 and 3 may be easily extended to cover the cases of 'thin' upper semicontinuous correspondences.

\section{Economic applications}

Much of the discussion of uniqueness in the economic literature was originally motivated by the theory of factor-price equalization in international trade theory. In the general model of two competitive economies with no joint production, constant returns to scale production functions, full employment and equal number of commodities and factors, commodity prices ( $p_{i}$ 's) and factor prices ( $w_{i}$ 's) in each country satisfy the relation $p=c(w), p, w \in \Omega$. The factor-price equalization theorem establishes that if certain conditions

Originally published in Journal of Mathematical Economics (1994) 23(5): 435-450. 
are fulfilled by the structure of the cost functions $c(w)$, then given any commodity price vector $(p)$, the factor price vector $(w)$, if it exists, must be unique. Samuelson (1953) sought to figure out these conditions by examining the Jacobian of the mapping $c(w)$. He thought of a sufficient condition which required that for any permutation of the rows and columns of the Jacobian, all its leading principle minors are nonvanishing. With a counter-example, Gale and Nikaido (1965) showed that this proposition paid insufficient attention to the domain of the mapping. They established that if the Jacobian has the property of P-matrix, then the factor-price equalization theorem holds. We have shown earlier (by giving an example) that a mapping has a unique solution although its associated Jacobian is neither a P-matrix nor a dominant diagonal matrix. Apart from the case of inflection, there is another interesting case of univalent mapping with vanishing Jacobian. Consider unit isocost curves drawn on the factor-price space. If the curves meet tangentially (the case of factor intensity reversal), the factor-price equalization theorem does not hold. On the other hand, it is possible that the curves always intersect but at the point of intersection two curves have the same slope. In this case the vanishing Jacobian does not affect univalency. In the former case, in the neighborhood of tangency, there must exist $w$ and $w^{\prime}$ such that eq. (6), presented in the next section, holds with the matrix of coefficients being singular. In this case the WPD condition is violated. In the second case, where univalency holds, the matrix of coefficients as in eq. (6) is nonsingular and the WPD condition holds. This shows that the WPD condition is more helpful in such cases than the Jacobian conditions. Now we state the factor-price equalization theorem in a suitable form.

Proposition 1 (factor-price equalization). Let $p=c(w), w, p \in \Omega$, represent the relationship between commodity prices and factor prices, i.e., a set of unit cost functions. Let $c(w)$ be a nondecreasing mapping. If $c(w)$ satisfies WPD condition, then for any assigned commodity price vector $(p)$, there exists a unique factor price vector $(w)$.

Now, if the vectors $w^{\prime}$ and $p^{\prime}$ are the relative prices of factors and commodities (respectively relative to the price of the factor $j$ and the price of commodity $k$ ) then $c_{i}\left(w^{\prime}\right)$ 's are not required to be linearly homogeneous and the Jacobian matrix of the system is not necessarily always nonnegative. In that case the use of the technique of P-matrix or dominant diagonal matrix requires knowledge beyond the sign-pattern of the Jacobian. However, utilizing Remark 3 together with Theorem 1, the following result is immediate.

Proposition 2 (relative factor-price equalization). Let $p^{\prime}=c\left(w^{\prime}\right), w^{\prime}, p^{\prime} \in \Omega$, represent the relationship between the relative commodity prices and the

Originally published in Journal of Mathematical Economics (1994) 23(5): 435-450. 
relative factor prices. If there exists a mapping $w^{\prime}=\Psi(q)$ which satisfies (4) such that $p^{\prime}=c(w)^{\prime} . \Psi=F(q)$ is a nondecreasing mapping and satisfies WPD condition then for any assigned relative commodity price vector $\left(p^{\prime}\right)$, there exists a unique relative factor price vector $\left(w^{\prime}\right)$.

The importance of the study of univalent mappings in economics has been established above all by the people who are interested in proving the uniqueness of competitive equilibrium. Let $z_{i}^{*}=z_{i}^{*}\left(p_{1}, \ldots, p_{n+1}\right), i=1, \ldots, n+1$, be a set of excess demand functions of a competitive economy, where $z_{i}^{*}$ stands for the excess demand for commodity $i$ and $\left(p_{1}, \ldots, p_{n+1}\right)$ represents the vector of commodity prices. It is well known that such excess demand functions are homogeneous of degree zero in prices.

A set of excess demand functions, $z_{i}^{*}\left(p_{1}, \ldots, p_{n+1}\right), i=1, \ldots, n+1$, is said to satisfy the property of weak gross substitute if and only if each $z_{i}^{*}$ is a nonincreasing function in $p_{i}$ and nondecreasing function in $p_{j}$ for $j=$ $1, \ldots, n+1$, and $j \neq i$ and $p \in \mathbb{R}^{n+1}$. Then an economy is said to be in competitive equilibrium whenever for a price vector $p \in \mathbb{R}^{n+1}, z^{*}(p)=0$. Now assuming $p_{n+1}=1$, let us normalize the price vector. The competitive equilibrium corresponding to a set of normalized excess demand functions, $z_{i}\left(p_{1}, \ldots, p_{n}\right), i=1, \ldots, n$, is said to be unique if the set of $n$ equations, $z_{i}\left(p_{1}, \ldots, p_{n}\right)=0$, has a unique solution vector. Then, following the technique in establishing Theorem 2, transform the normalized excess demand functions to

$$
z_{i}=R_{i}\left(t_{1 i}, \ldots, t_{n i}\right), \quad i=1, \ldots, n,
$$

where $t_{j i}=p_{i}-p_{j}$ and $t_{i i}=p_{i}$. Since $R_{i}$ satisfies the property of weak gross substitute and also since $t_{j i}^{\prime}$ 's are normalized, $R_{i i}$ must be a nonincreasing function. Now the result below is immediate.

Proposition 3. If the set of excess demand functions of a competitive economy, $z_{i}^{*}\left(p_{1}, \ldots, p_{n+1}\right), i=1, \ldots, n+1$, satisfies the property of weak gross substitute, then the normalized mapping, $z_{i}\left(p_{1}, \ldots, p_{n}\right), i=1, \ldots, n$, is univalent if and only if $z\left(p_{1}, \ldots, p_{n}\right)$ satisfies $W N D$.

\section{Proof of the theorems}

Before proving the univalency results first consider a set of nondecreasing functions $y_{i}=f_{i}\left(x_{1}, \ldots, x_{n}\right), i=1, \ldots, n$, which satisfies WPD. Now we describe the procedure of constructing an $n \times n$ matrix in $p_{i j}$ 's corresponding to the given set of mappings. For any assigned values $y_{1}, y_{2}, \ldots, y_{n}$ if there exist two solution vectors $x$ and $x^{\prime}$ define $t_{i i}=x_{i}, t_{j i}=\left(x_{j}+x_{i}\right)$ for $j \neq i$ and $t_{i i}^{\prime}=x_{i}^{\prime}$, $t_{j i}^{\prime}=\left(x_{j}^{\prime}+x_{i}^{\prime}\right)$ for $j \neq i$.

First consider the case for $t_{11} \leq t_{11}^{\prime}$. Then, by definition,

Originally published in Journal of Mathematical Economics (1994) 23(5): 435-450. 


$$
\begin{aligned}
0= & P_{1}\left(t_{11}^{\prime}, \ldots, t_{n 1}^{\prime}\right)-P_{1}\left(t_{11}, \ldots, t_{n 1}\right) \\
= & {\left[P_{1}\left(t_{11}^{\prime}, \ldots, t_{n 1}^{\prime}\right)-P_{1}\left(t_{11}, t_{21}^{\prime}, \ldots, t_{n 1}^{\prime}\right)\right]+\left[P_{1}\left(t_{11}, t_{21}^{\prime}, \ldots, t_{n 1}^{\prime}\right)\right.} \\
& \left.-P_{1}\left(t_{11}, t_{21}, t_{31}^{\prime}, \ldots, t_{n 1}^{\prime}\right)\right]+\cdots+\left[P_{1}\left(t_{11}, \ldots, t_{(n-1) 1}, t_{n 1}^{\prime}\right)\right. \\
& \left.-P_{1}\left(t_{11}, \ldots, t_{n 1}\right)\right] .
\end{aligned}
$$

Define, for $t_{j 1}^{\prime} \neq t_{j 1}$,

$$
p_{1 j}=\left[P_{1}\left(t_{11}, \ldots, t_{(j-1) 1}, t_{j 1}^{\prime}, \ldots, t_{n 1}^{\prime}\right)-P_{1}\left(t_{11}, \ldots, t_{j 1}, t_{(j+1) 1}^{\prime}, \ldots, t_{n 1}^{\prime}\right)\right] . d
$$

where $d=\left(t_{j i}^{\prime}-t_{j i}\right)^{-1}$; otherwise, for $t_{j 1}^{\prime}=t_{j 1}, p_{1 j}=1$. Since $t_{j 1}^{\prime} \geq t_{11}^{\prime} \geq t_{11}$ for all $j, p_{1 j}$ 's are defined for $x, y \in S$.

Now consider the case for $t_{11} \geq t_{11}^{\prime}$. Define, for $t_{j 1}^{\prime} \neq t_{j 1}$,

$$
p_{1 j}=\left[P_{1}\left(t_{11}^{\prime}, \ldots, t_{(j-1) 1}^{\prime}, t_{j 1}, \ldots, t_{n 1}\right)-P_{1}\left(t_{11}^{\prime}, \ldots, t_{j 1}^{\prime}, t_{(j+1) 1}, \ldots, t_{n 1}\right)\right] . d^{*},
$$

where $d^{*}=\left(t_{j 1}-t_{j 1}^{\prime}\right)^{-1}$; otherwise, for $t_{j 1}^{\prime}=t_{j 1}, p_{1 j}=1$. Now rewrite (5) as

$$
\begin{aligned}
0= & {\left[P_{1}\left(t_{11}, \ldots, t_{n 1}\right)-P_{1}\left(t_{11}^{\prime}, \ldots, t_{n 1}^{\prime}\right)\right] } \\
= & {\left[P_{1}\left(t_{11}, \ldots, t_{n 1}\right)-P_{1}\left(t_{11}, \ldots, t_{(n-1) 1}, t_{n 1}^{\prime}\right)\right] } \\
& +\left[P_{1}\left(t_{11}, \ldots, t_{(n-1) 1}, t_{n 1}^{\prime}\right)-P_{1}\left(t_{11}, \ldots, t_{(n-1) 1}^{\prime}, t_{n 1}^{\prime}\right)\right] \\
& +\cdots+\left[P_{1}\left(t_{11}, t_{21}^{\prime}, \ldots, t_{n 1}^{\prime}\right)-P_{1}\left(t_{11}^{\prime}, \ldots, t_{n 1}^{\prime}\right)\right] .
\end{aligned}
$$

By WPD, $p_{1 j} \geq 0$. If we define $\delta x=x^{\prime}-x$, then $t_{j 1}^{\prime}-t_{j 1}=\delta x_{j}+\delta x_{1}$ for all $j \neq 1$ and $t_{11}^{\prime}-t_{11}=\delta x_{1}$. Using the definition of $p_{1 j},(5)$ may be rewritten as

$$
0=p_{11} \delta x_{1}+p_{12}\left(\delta x_{2}+\delta x_{1}\right)+\cdots+p_{1 n}\left(\delta x_{n}+\delta x_{1}\right) .
$$

Similarly $p_{i j}$ 's for all $i, j=1,2, \ldots, n$, may be defined, which following the construction of $\left(5^{\prime}\right)$ from $(5)$ gives

$$
\begin{aligned}
& 0=p_{11} \delta x_{1}+p_{12}\left(\delta x_{2}+\delta x_{1}\right)+\cdots+p_{1 n}\left(\delta x_{n}+\delta x_{1}\right), \\
& 0=p_{21}\left(\delta x_{1}+\delta x_{2}\right)+p_{22} \delta x_{2}+\cdots+p_{2 n}\left(\delta x_{n}+\delta x_{2}\right), \\
& \vdots \\
& 0=p_{n 1}\left(\delta x_{1}+\delta x_{n}\right)+\cdots+p_{n, n-1}\left(\delta x_{n-1}+\delta x_{n}\right)+p_{n n} \delta x_{n} .
\end{aligned}
$$

Originally published in Journal of Mathematical Economics (1994) 23(5): 435-450. 
Alternatively, we can write (6) in vector matrix form as

$$
\left[\begin{array}{c}
0 \\
0 \\
\vdots \\
0
\end{array}\right]=\left[\begin{array}{cccc}
\sum_{j=1}^{n} p_{1 j} & p_{12} & \ldots & p_{1 n} \\
p_{21} & \sum_{j=1}^{n} p_{2 j} & \ldots & p_{2 n} \\
\vdots & \vdots & & \vdots \\
p_{n 1} & p_{n 2} & \ldots & \sum_{j=1}^{n} p_{n j}
\end{array}\right]\left[\begin{array}{c}
\delta x_{1} \\
\delta x_{2} \\
\vdots \\
\delta x_{n}
\end{array}\right] .
$$

Note that the matrix of the right-hand side would be equivalent to the Jacobian of the mappings $y_{i}=f_{i}\left(x_{1}, \ldots, x_{n}\right), i=1, \ldots, n$, whenever for each $i, f_{i}$ is nondecreasing and differentiable with respect to all its arguments (i.e., $\delta f_{i} / \delta x_{j} \geqq 0$ for all $i$ and $\left.j\right)$, since for $i, j=1, \ldots, n,\left(\delta f_{i} / \delta x_{j}\right)=p_{i j}$ for $i \neq j$ and $\left[\left(\partial f_{i} / \partial x_{i}\right)-\sum_{j \neq i}\left(\delta f_{i} / \delta x_{j}\right)\right]=p_{i i}$.

\section{Proof of Theorem 1}

To the contrary, suppose for some assigned value of $y$, the solution vector $x$ exists and is not unique. Assume, $x$ and $x^{\prime}$ are two solution vectors. Construct $p_{i j}$ 's in the way explained earlier. By definition, (6) is true. We shall complete our proof by demonstrating that, $\delta x_{1}=\delta x_{2}=\cdots=\delta x_{n}=0$.

Suppose $\delta x_{i} \neq 0$ for some $i$. Without any loss of generality assume $\delta x_{i}>0$. We shall show that there must exist some $\delta x_{j}$ such that $\left|\delta x_{j}\right|>\left|\delta x_{i}\right|$. Consider the $i$ th equation of system (6):

$$
0=p_{i 1}\left(\delta x_{1}+\delta x_{i}\right)+\cdots+p_{i i} \delta x_{i}+\cdots+p_{i n}\left(\delta x_{n}+\delta x_{i}\right) .
$$

If $p_{i i}>0, p_{i i} \delta x_{i}>0$. By WPD, $p_{i j} \geqq 0$. Hence there exists a negative $\delta x_{j}$ such that $\left|\delta x_{j}\right|>\left|\delta x_{i}\right|$. If $p_{i i}=0$, by WPD, there exists an element $a$ in the index set such that $P_{i a}$ is an increasing function of $t_{a i}$ which also implies that $P_{i a}$ is an increasing function of $x_{a}$. If $\delta x_{i} \neq \delta x_{a}$ then $p_{i a}>0$. Suppose $\delta x_{i}<-\delta x_{a}$. Obviously our search for a $\delta x_{j}$, such that $\left|\delta x_{j}\right|>\left|\delta x_{i}\right|$, ends with the choice of $j=a$. Again if $\delta x_{i}>-\delta x_{a}$, since $p_{i i} \delta x_{i}=0$ and $p_{i a}\left(\delta x_{a}+\delta x_{i}\right)>0$, by (7) there exists some $\delta x_{j}$ which is negative and $\left|\delta x_{j}\right|>\left|\delta x_{i}\right|$.

The problem arises when $p_{i i}=0$ and $-\delta x_{a}=\delta x_{i}$. Then consider the relation (a) of (6):

$$
0=p_{a 1}\left(\delta x_{1}+\delta x_{a}\right)+\cdots+p_{a a} \delta x_{a}+\cdots+p_{a n}\left(\delta x_{n}+\delta x_{a}\right) .
$$

Again, by WPD, either there exists a positive $\delta x_{j}$ such that $\left|\delta x_{j}\right|>\left|\delta x_{a}\right|=\left|\delta x_{i}\right|$ or there exists $b$ in the index set such that $\delta x_{b}$ is positive and $\left|\delta x_{b}\right|=\left|\delta x_{a}\right|=$

Originally published in Journal of Mathematical Economics (1994) 23(5): 435-450. 
$\left|\delta x_{i}\right|$. In the former situation our search for a $\delta x_{j}$ such that $\left|\delta x_{j}\right|>\left|\delta x_{i}\right|$ ends. In the latter situation we proceed to consider the relation (b) of (6):

$$
0=p_{b 1}\left(\delta x_{1}+\delta x_{b}\right)+\cdots+p_{b b} \delta x_{b}+\cdots+p_{b n}\left(\delta x_{n}+\delta x_{b}\right) .
$$

By WPD this search must end. In case of worst luck we have to continue till we reach a situation where, $\left|\delta x_{k}\right|=\left|\delta x_{g}\right|=\cdots=\left|\delta x_{a}\right|=\left|\delta x_{i}\right|$. By WPD again $p_{k \boldsymbol{k}}>0$. Also,

$$
0=p_{k 1}\left(\delta x_{1}+\delta x_{k}\right)+\cdots+p_{k k} \delta x_{k}+\cdots+p_{k n}\left(\delta x_{n}+\delta x_{k}\right) .
$$

Depending on whether $\delta x_{k}$ is positive or negative now there must exist a negative or positive $\delta x_{j}$ such that $\left|\delta x_{j}\right|>\left|\delta x_{k}\right|=\cdots=\left|\delta x_{i}\right|$ to satisfy the above equation.

We have arrived at the conclusion that if $\delta x_{i}>0$ for some $i$, there exists $\delta x_{j}$ such that $\left|\delta x_{j}\right|>\left|\delta x_{i}\right|$. It is clear from the nature of our argument that if $\delta x_{i}<0$ from some $i$, there would also exist some $\delta x_{j}$ with $\left|\delta x_{j}\right|>\left|\delta x_{i}\right|$. Now, suppose $\delta x=x^{\prime}-x \neq 0$. Consider a $\delta x_{m}$ such that $\left|\delta x_{m}\right| \geq\left|\delta x_{i}\right|$ for all $i$. We have just shown above that WPD requires the existence of a $\delta x_{k}$ such that $\left|\delta x_{k}\right|>\left|\delta x_{m}\right|$ which contradicts the choice of $\delta x_{m}$. Therefore, $\delta x=x^{\prime}-x=0$, which proves our theorem.

Q.E.D.

\section{Proof of Theorem 3}

Given Theorem 2, we have to establish only the necessity part. Suppose, WND, is not satisfied in the cell $\left(x_{0} \pm k\right)$ where $k$ is a positive $n$-dimensional vector $\left(k_{1}, \ldots, k_{n}\right)$. Since $f(x)$ is continuous almost everywhere, pick up $x \in\left(x_{0} \pm k\right)$, such that $f(x)$ is continuous in the neighborhood of $x$, the chain condition breaks down. We can divide $y=f(x)$ into two sets of functions. The set $I$ contains those functions which satisfy the chain condition and the set $J$ contains those functions which do not satisfy the chain condition. Note, for $i \in J, P_{i i}$ and $P_{i j}(i \neq j$ and $j \notin J)$ are locally invariant in the neighborhood of $x$. Therefore, in the neighborhood of $x$, we may write, $y_{i}=P_{i}\left(t_{j i}, \ldots, t_{n i}\right)$, $i=j, \ldots, n$, where $t_{i i}=x_{i}$ and $t_{k i}=x_{i}-x_{k}$. Since $P_{i}$ is invariant with respect to $t_{i i}$, for $i \in J$, and $t_{k i}$ is the difference of two terms, $y_{i}(i \in J)$ remains invariant if we add a small value $\delta>0$ to each element of the solution vector. That is, if $x_{i}(i \in J)$ is a solution vector for $y_{i}=f\left(x_{j}, \ldots, x_{n}\right), i \in J$, then $\left(x_{j}+\delta\right)$ is also a solution vector. Let $x_{i}^{\prime}(i \in I)$ be such that $y_{i}=\left(x_{1}^{\prime}, x_{2}^{\prime}, \ldots, x_{j-1}^{\prime}, x_{j}+\delta \ldots, x_{n}+\delta\right)$ for $i \in J$. Remember, $\delta$ is an arbitrary but small positive number and $y_{\mathrm{i}}$ is a continuous function in the neighborhood of $x$ satisfying WND with respect to the variables $\left(x_{1}, \ldots, x_{j-1}\right)$. Hence, the solution $x_{i}^{\prime}(i=1, \ldots, j-1)$ exists and satisfies a vector-matrix relationship similar to eq. $\left(6^{\prime}\right)$ in section 3 . It is clear that if $x=\left(x_{1}, \ldots, x_{n}\right)$ is a solution vector then $x^{\prime}=\left(x_{1}^{\prime}, \ldots, x_{j-1}^{\prime}\right.$, $x_{j}+\delta, \ldots, x_{n}+\delta$ ) is also a solution vector for $y=f(x)$. Q.E.D.

Originally published in Journal of Mathematical Economics (1994) 23(5): 435-450. 


\section{References}

Arrow, K.J. and F. Hahn, 1971, General competitive analysis (Holden-Day, San Francisco, CA).

Chipman, J.S., 1964, Factor price equalization and the Stolper-Samuelson Theorem (abstract), Econometrica 32, 682-683.

Duffin, R.K., 1948, Nonlinear networks, $\Pi^{\text {b}}$, American Mathematical Society Bulletin 54, $119-127$.

Gale, D. and H. Nikaido, 1965, The Jacobian matrix and global univalence of mappings, Mathematische Annalen 159, 81-93.

Hicks, J.R., 1946, Value and capital (Oxford University Press, London).

Hiriart-Urruty, J.B.E., 1981, A better insight into the generalized gradient of the absolute value of a function, Applicable Analysis 12, 239-249.

Hiriart-Urruty, J.B.E., 1982, Characterization of the plenary hull of the generalized Jacobian matrix, Mathematical Programming Study 8, 1-12.

Kehoe, T., 1980, An index theorem for general equilibrium models with production, Fconometrica 48, 1211-1232.

Levhari, D., J. Paroush and B. Peleg, 1975, Efficiency analysis of multivariate distributions, Review of Economic Studies 42, 87-91.

Mas-Colell, A., 1970, Homeomorphisms of compact, convex sets and the Jacovian matrix, SIAM Journal of Mathematical Analysis 10.

Mas-Colell, A., 1979, Two propositions on the global univalence of systems of cost functions, in: J.R. Green and J.A. Scheinkman, eds., General equilibrium, growth, and trade (Academic Press, New York).

Mas-Colell, A., 1985, The theory of general equilibrium (Cambridge University Press, Cambridge, UK).

McKenzie, L.W., 1959, Matrices with dominant diagonal and economic theory, in: K.J. Arrow, S. Karlin and P. Suppes, eds., Mathematical methods in social sciences (Stanford University Press, Stanford, CA).

Nikaido, H., 1968, Convex structures and economic theory (Academic Press, New York).

Pearce, I. and J. Wise, 1973, On the uniqueness of competitive equilibirum: Part I, unbounded demand, Econometrica 41, 817-826.

Pearce, I. and J. Wise, 1974, On the uniqueness of competitive equilibrium: Part II, bounded demand, Econometrica 42, 921-932.

Samuelson, P.A., 1953, Prices of factors and goods in general equilibrium, Review of Economic Studies $21,1-20$. 